\title{
Using psychotherapy effectively: choosing an appropriate treatment
}

\author{
Richard Tillett
}

Making effective use of psychotherapy can be difficult for patients and their doctors, for a number of reasons. First, the term 'psychotherapy' itself is unsatisfactory, used by some people to indicate a particular type of psychotherapy (usually psychodynamic), and by others to embrace all forms of psychological treatment. When we use the term 'medication' we usually add a qualifying adjective (e.g. antidepressant or antipsychotic medication) and it is helpful to do so in psychotherapy (e.g. dynamic therapy, cognitive therapy, etc.).

There is a bewildering array of approaches (over 400 types of psychotherapy have been described) each with its own theoretical basis and technique, offering a range of complexity and sophistication, yet all apparently of equivalent effectiveness.

Psychotherapy is not an exact science; most approaches recognise the crucial importance of the therapeutic relationship, a human interaction which is difficult to evaluate scientifically. This contributes to the notorious difficulty of psychotherapy research, especially in those approaches which focus on the treatment relationship (e.g. dynamic and humanistic psychotherapies). Historically, psychotherapists have been unenthusiastic about research because the reductionism of scientific method conflicts with recognition of the subtleties and complexities of human relationships.

We are often unscientific in our approach to treatment; doctors and patients are both liable to be attracted to some approaches rather than others in the light of our own beliefs and personal experiences.

The availability of psychological treatments across the UK is extremely variable so that treatment is often determined by the availability of local resources rather than an appropriately needs-led assessment.

In helping a patient to find an appropriate treatment we need to have some knowledge of the range and types of treatment available (and the evidence of their efficacy) and a detailed understanding of the patient for whom the treatment is intended. Given that all psychotherapies involve active collaboration between patient and therapist, it follows that assessment and treatment planning should also be a collaborative process (Tillett, 1996). Both common sense and research evidence suggest that patients are more likely to participate actively in treatments which they have helped to plan. This paper is intended to offer a simple and practical guide for the busy clinician.

\section{The evidence base for psychotherapy}

Over the past 40 years, research has established that, in general, psychological treatments are effective. Meta-analytical studies (Smith \& Glass 1977; Smith et al, 1980; Shapiro \& Shapiro, 1982; Lipsey \& Wilson, 1993) consistently show that psychological treatments produce effect sizes of $0.8-1.0$ (equivalent to antidepressant medication, for example). Specific efficacy has been demonstrated in many different situations: for example, family intervention has been shown to reduce relapse and readmission rates in schizophrenia (Leff, 1985; Lam, 1991); cognitive therapy is an effective treatment for depression and may confer greater prophylactic benefit than medication (Williams, 1992); cognitive-behavioural therapy is effective in eating disorders (Hartmann et al, 1992); behavioural therapy is more effective than medication in the treatment of phobic disorder (Marks, 1981); and dynamic psychotherapy is effective in borderline personality disorder (Higgitt \& Fonagy, 1992; Stevenson \& Meares, 1992). 
Differential efficacy has proved more resistant to research evaluation but there are some suggestions that structured behavioural and cognitive therapies produce greater effect sizes than dynamic and humanistic approaches (Smith et al, 1980; Shapiro \& Shapiro, 1982, Svartberg \& Stiles, 1991; CritsChristoph, 1992). However, these advantages rarely reach statistical significance and many studies are of short-term therapy where focused and structured approaches might be expected to show an advantage. There is some evidence that patients with certain psychological traits will respond to specific forms of psychotherapy (Beutler, 1991), and some evidence of a dose-response relationship at least in the early stages of therapy (Orlinsky \& Howard, 1993, Clementel-Jones et al, 1990).

In 1996, the Department of Health undertook a major review of the provision of psychotherapy services in England, which included a national survey of treatment resources and a major study of the evidence base for psychotherapeutic treatment. The survey confirmed the disorganisation of service provision with widespread confusion about what psychotherapy is, who should provide it and for whom. It also confirmed the patchy and inconsistent distribution of services and the lack of effective coordination and service-planning both locally and nationally (Parry \& Richardson, 1996). The review of the research literature demonstrated a sound evidence-base for psychotherapy in general, but confirmed the relative lack of scientific research in the areas of dynamic and creative therapies (Roth \& Fonagy, 1996). Both parts of the review affirmed the case for needs-led, evidence-based, patient-focused treatment, subject to appropriate supervision and audit and available on an equitable basis across the country. The review also examined implications for training organisations and areas for further research.

\section{Know your patient}

It is not possible to make an intelligent recommendation about psychological treatment without detailed knowledge of the patient and his or her circumstances (see Box 1). This will include, first, a careful account of the presenting problem; detailed exploration is necessary of the circumstances in which the problem first appeared, factors which appear to aggravate or mitigate it, and associated behavioural responses, thoughts and feelings. Second, what attempts have been made by the patient to solve their problems and what help or treatment has been offered by others? Third, one needs a detailed account of the patient's background, both past and present; a biographical
Box 1. Know your patient

Presenting problem (details)

Solutions/treatment already tried

Background history

Current circumstances

Mental state examination

Attitude to treatment

Family/couple dynamics

narrative is useful in piecing together a developmental history but current circumstances and relationships are equally important. Fourth, as well as an orthodox mental state examination, one needs to explore the patient's attitude to treatment. Many patients expect a conventional medical consultation in which a treatment will be chosen and prescribed by the doctor. However gratifying for both patient and doctor, this is not an appropriate basis for psychotherapy. One needs to explore patients' abilities to recognise some responsibility for their problems and their willingness to work actively towards finding solutions. If one of the more sophisticated therapies is being considered, one also needs to explore whether the patient can make use of psychological insight and has the capacity (and willingness) to enter into an intensive treatment relationship. The way in which the assessment interview unfolds and the subjective responses of the interviewer are also helpful in anticipating the possible course of treatment. Fifth, if the patient lives in close relationship with one or more others (e.g. as a couple or family), it is essential to meet with them also (both separately and with the patient) to explore the interrelationship between the patient's problems and the social system in which he or she lives. Failure to explore family dynamics is one of the most common deficiencies in psychotherapy treatment planning.

\section{Understanding the problem - formulation}

Most clinical problems can be understood from a number of theoretical perspectives. In helping patients to choose an appropriate treatment it is helpful to offer tentative explanations (formulations) of their problems to see which makes most sense to them (see Box 2). This will lead naturally to an explanation of how different approaches work and form a sound basis for collaborative treatment planning. The discussion of different explanatory 
Box 2. Formulating the problem

Behavioural

Cognitive

Dynamic

Systemic models also offers patients alternative ways of understanding their problems and is likely to be therapeutic in itself. For example, patients who see their problems in medical terms may find a psychological explanation equally convincing, allowing them to think afresh about their problems and opening up a wider range of treatment possibilities. Different types of psychological formulation offer alternative explanatory models and lead naturally towards different types of psychotherapy.

A behavioural formulation will focus on the antecedents and consequences of a problematic behaviour and will provide an explanation based on learning theory (Marks, 1981). A cognitive formulation will seek to explain the difficulty in terms of characteristic types of maladaptive thinking, recognising that these may be recently acquired or long-standing schemata (Williams, 1992). A dynamic formulation will seek to establish links between patients' current problems, their unconscious psychological processes and their early life experiences (Aveline, 1980). A common basis for dynamic formulation is provided by the 'two triangles' elaborated by Malan (1976). A systemic formulation will explore the possible meaning or effect of the patient's symptoms in terms of the interrelationships within a couple or family system (Barker, 1992).

\section{Treatment planning and contract}

In an age of managed health care, it is no longer appropriate to embark hopefully on psychotherapy without a clear treatment plan. As in other areas of practice, application of the Care Programme Approach forces us to be more specific about what we are trying to achieve and the means of doing so. There are a number of principles which underly sound psychotherapeutic treatment planning (see Box 3 ).

One should use the minimum necessary treatment to achieve progress. Many psychotherapists and their patients are attracted by the idea of doing the job thoroughly, but this may occupy scarce treatment resources for long periods and reduce the number
Box3. Principles of treatment planning

Least necessary intervention

Collaborative process

Based on formulation

Meet (\& treat?) family

Provisional treatment contract

of patients who can be seen. In general, patients with specific problems of short duration can be treated with brief psychotherapies, often by non-specialist personnel. Patients with more intractable problems may need longer-term, more intensive psychotherapy, probably from a member of a specialist team.

Treatment planning should be based on a collaborative approach, out of respect for the patient, and to maximise motivation and commitment.

It should also be based on sound assessment and formulation, not chosen (by doctor or patient) simply from personal preference.

Unless the patient lives in isolation, one should consider the possibility of systemic therapy. Personal experience suggests that it is always worth meeting, and often worth treating, the couple or family as a unit.

After assessment, one should be able to agree a provisional treatment contract with the patient. This has the advantage of clarifying for both parties what has been agreed, and it complies with the requirements of care programming. It also provides a containing function for both client and therapist, but must be open to renegotiation if the original assessment proves to be inaccurate. Some patients and therapists dislike treatment contracts, preferring an open-ended approach, but personal experience suggests that treatment contracts encourage focused thinking (by both therapist and patient) and help to reduce overall treatment duration.

\section{Key questions}

In helping a patient to decide an appropriate treatment plan we need to answer several key questions (see Box 4).

\section{Is any treatment required?}

The fact that a patient seeks treatment does not necessarily mean that treatment is indicated or will be helpful. Some patients will consult for problems 
Box 4. Key questions

Is any treatment required?

(Relative merits of) medical/ psychological/ social treatment?

What type of psychotherapy?

What depth (dose) of therapy?

Who should be involved?

they could solve themselves or will seek help for problems that are insoluble. Help-seeking behaviour is often motivated by 'attachment hunger' - the patient is seeking human contact rather than professional treatment. Providing treatment may gratify both doctor and patient, but may also set up a collusion which becomes dependent and difficult to end. It is always worth considering the option of no treatment.

\section{If treatment is indicated, what are the relative merits of different interventions?}

As trainees we are taught to approach treatment planning by looking at three main areas of intervention - medical, psychological and social. As doctors we are prone to overvalue medical interventions at the expense of psychological and social approaches. Medical approaches are, however, often effective, less time-consuming and widely available. Psychologically minded patients (and doctors) may naturally incline towards a psychotherapeutic approach, but this may not be the most effective or cost-beneficial treatment. Social interventions are readily overlooked because neither patient nor professional thinks of them as treatment and they are often dependent on the patient taking action him- or herself. Careful treatment planning will always involve looking at costs and likely benefits of a range of interventions.

\section{Which types of psychotherapy might be appropriate?}

Most patients are potentially able to benefit from a number of different psychological treatments. Which to choose for a particular patient will depend on his or her unique circumstances and symptoms. Motivation for treatment is likely to be enhanced if the patient makes an informed decision in collaboration with the assessor, discussing the different approaches available and their likely advantages and disadvantages.

\section{What depth of therapy is needed?}

In general, it is sensible to offer the minimum necessary intervention unless it is clear that a more comprehensive approach is required. In general, patients with circumscribed problems and sound personalities will make effective use of simple, brief interventions. Patients with diffuse or chronic problems will require more substantial interventions, although much can be achieved by the use of focused therapy, even with patients with significant personality disturbance. Within the National Health Service, long-term psychodynamic psychotherapy will usually be reserved for patients with chronic and intractable disorders. Assessment of the 'dose' of psychotherapy should form part of every psychological treatment proposal.

\section{Who should therapy involve?}

For many patients, individual treatment is appropriate, but patients and their doctors are both liable to overlook the potential benefits of family or group therapy. Patients living in close domestic relationships should be seen with their significant others so that an informed view can be achieved of whether systemic therapy would be helpful (or perhaps even essential). Experience in my own department suggests that the use of systemic therapy is effective and may significantly reduce treatment duration. Group psychotherapy may be helpful in treating homogeneous groups of patients with specific problems (e.g. anxiety and eating disorders), whereas heterogeneous groups have specific advantages over individual therapy (Yalom, 1985) and enable a single therapist to work with several patients simultaneously.

\section{A hypothetical patient}

Carol is a 32-year-old nurse, married to John (41), an electrical engineer. They have two children: Hannah aged 10 and Ben aged 7. Carol presents with a 10-year history of recurrent depressive and anxiety symptoms.

The first episode occurred three months after Hannah's birth and responded to antidepressant medication from her general practitioner. Subsequent episodes have tended to be more prolonged and have responded less well to antidepressant medication. She was hoping to return to work part-time when the children were of school age, but when she attempted this she could not cope with the stress at work and became increasingly depressed.

At the time of presentation she is low in mood with occasional suicidal ideas about which she feels ashamed and guilty. Her self-esteem is obviously low 
and she is angry with herself for not coping better. She is reluctant to go out on her own except to collect the children from school, and has lost interest in sex. Her relationship with John has become strained; he has had to take time off from work to support the home situation and feels that his wife's lack of interest in sex means that she no longer loves him.

Carol was born and brought up locally, the eldest of three siblings, and her parents still live nearby (as do John's). Her father, an unskilled labourer, was prone to drinking and gambling. Her mother had rheumatoid arthritis and was increasingly disabled. As the eldest child, Carol did a lot to help her mother with the cooking, cleaning and shopping. John was also brought up locally; his father was a successful businessman but his mother suffered from recurrent depression, although she was never hospitalised because of this.

At interview, Carol presents as a rather tiredlooking woman in her early 30 s, obviously tense and occasionally tearful especially when talking about her inability to cope. She is keen to have help, but seems to have little idea about what form this might take and is happy to let someone else decide.

\section{Formulating the problem psychologically}

In Carol's case, a behavioural formulation might focus on the specific difficulty of her returning to work, seeing this in terms of an anxiety to which she could be desensitised. A cognitive formulation might focus on the way in which her habits of thinking are reinforcing her low mood and poor selfesteem. A dynamic formulation would suggest that her current difficulties might be related to unconscious feelings (of hostility for example), which were generated originally by the unsatisfactory circumstances of her childhood, and which have been repressed through denial and the adoption of a caring role both professionally and in her family. A systemic formulation might look at the significance of her present symptoms in terms of her relationship with her husband or parents (or both). None of these explanatory models is correct; all are possible and they are not mutually exclusive.

\section{Helping Carol to choose a treatment}

We can do this by working through the key questions outlined previously.

\section{Is treatment of any kind required?}

At first sight, yes. Carol is obviously depressed, her family are concerned and her general practitioner is seeking advice. The temptation to 'do something' is obvious. The possibility exists, however, that professional help will impair her already damaged selfrespect and collude with her passivity. She could be encouraged to develop ideas of her own about how the situation should be tackled, which would lessen her dependency and improve her self-esteem. A joint interview with her husband (as part of the assessment process) might enable them to explore how they could tackle the problem together, maximising their joint resources and reducing the emphasis on Carol as the identified patient. Treatment could then be deferred for a suitable period to see how they get on.

What are the relative merits of medical, psychological and social intervention?

A medical approach would examine her previous treatment (and her compliance with it), would look for organic or iatrogenic causes of prolonged depression (for example, hypothyroidism or antihypertensive medication), would probably explore the use of further antidepressant medication with or without augmentation therapy, and might include the possibility of electroconvulsive therapy.

Psychological interventions are explored below. Social interventions might include: support from a community keyworker; provision of help with child care and domestic tasks; attendance at a day centre; and help with finding satisfying occupations other than work.

\section{Which types of psychotherapy might be appropriate?}

Carol's case offers a wide variety of possible interventions. Her anxiety and phobic symptoms might respond to behavioural therapy, and the resulting improvement in her self-esteem might help to lift her mood. She could use a cognitive approach to look at the depressed and anxious maladaptive thinking patterns that have become established. Psychodynamic therapy would help her to explore the links between her current problems and the difficulties in her childhood. A systemic approach would evaluate her relationship with her husband, her children and her parents, all of whom are closely involved in her daily life. Each may have ideas about how the situation might be changed. Each (or all) of these may unwittingly be reinforcing the problem by attempting to help.

\section{What depth of therapy is needed?}

A brief intervention may be sufficient to unlock the stuck situation; this has the benefit of economy and allowing Carol to sort out most of the problems for herself. It should, therefore, be tried first. If, however, simple interventions have already been tried, a more 
sophisticated approach may be needed. In Carol's case, this might involve individual psychodynamic psychotherapy.

\section{Who should therapy involve?}

Carol sees the problem as hers and, to some extent, so do her family. This may be part of the problem. Assessment should certainly involve her husband to explore the dynamics of their relationship and to assess their willingness to work jointly to solve the problem. Assessment might also include the children or, more probably, all Carol's and John's extended families. In practice, couple therapy is both effective and feasible and is usually the obvious choice. In this case, it would enable Carol and John to work together on their problems, reducing the risk of dependency on their families of origin or on their own children. If, however, it becomes clear that other family members (for example Carol's parents) are likely to remain entangled in the situation, family therapy may be necessary.

Assessment in this case would probably involve seeing Carol individually, then with her husband/ parents before a discussion of the different formulations and treatment options.

\section{Negotiating a treatment contract}

By the end of assessment, we have a considerable amount of information about Carol, her problems and her background. We also have some idea of how she thinks about the problem and her attitude towards different forms of treatment. We also know something of her relationship with John and other members of the extended family and their likely attitudes towards treatment. During assessment, Carol and John will have formed their own impressions of the different types of treatment available. We are therefore ready to negotiate a treatment contract. At this stage, they may try to leave the decision to the doctor (and the doctor may be tempted to decide), or they may opt quickly for a particular approach because it fits in with their existing ideas (or enables them to avoid awkward topics). Their doctor would be wise to insist gently on discussing all the options before helping them to make a decision.

In Carol's case, most psychiatrists would want to review her medical treatment as a first priority. But noting that no previous attempt has been made at psychological treatment, and in view of its proven efficacy, psychological treatment would also seem worth considering. Applying the principle of 'the least necessary treatment', a short-term cognitive or behavioural intervention would be the logical first choice. If this is unsuccessful, one could review treatment with Carol and her husband, looking perhaps at the possibility of either dynamic or systemic therapy. If it seems that couple or family processes are reinforcing Carol's depression and helplessness, systemic therapy would probably be quicker and more effective in achieving change.

\section{Conclusion}

The effective use of psychotherapy requires an understanding of the different approaches available and their potential application to a particular clinical situation. In an ideal world, all patients would have a detailed assessment before treatment is recommended; in practice, this is not always possible. It is, however, unacceptable (and ineffective) to recommend psychological treatment without careful assessment, including a discussion of the different options with the patient so that a properly informed decision can be made. Many patients, especially those with short-term and specific problems can be helped by brief psychological interventions, often delivered by non-specialist personnel. Patients with chronic or pervasive problems may benefit from brief therapies if a focus can be found for treatment, but may need more extended and sophisticated treatment for which more detailed assessment is required. The simpler forms of psychological treatment are widely available in most districts; specialist services are thinly and unevenly distributed. If you need advice about a difficult case, talk to your local consultant psychotherapist.

\section{References}

Aveline, M. (1980) Making a psychodynamic formulation. Psychiatric Bulletin, 4, 192-193.

Barker, P. (1992) Basic Family Therapy (3rd edn). Oxford: Blackwell.

Beutler, L. E. (1991) Selective treatment matching: systematic eclectic psychotherapy. Psychotherapy, 28, 457-462.

Clementel-Jones, C., Malan, D. \& Trauer, T. (1990) A retrospective follow-up study of 84 patients treated with individual psychoanalytic psychotherapy: outcome and predictive factors. British Journal of Psychotherapy, 6, 363-374.

Crits-Christoph, P. (1992) The efficacy of brief dynamic psychotherapy: a meta-analysis. American Journal of Psychotherapy, 149, 151-158.

Hartmann, A., Herzog, T. \& Drinkmann, A. (1992) Psychotherapy of bulimia: what is effective? A metaanalysis. Journal of Psychosomatic Research, 36, 159-167.

Higgitt, A. \& Fonagy, P. (1992) Psychotherapy in borderline and narcissistic personality disorder. British Journal of Psychiatry, 161, 23-43.

Lam, D. (1991) Psycho-social family interventions in schizophrenia: A review of empirical studies. Psychological Medicine, 21, 423-441. 
Leff, J. (1985) Family treatment of schizophrenia. In Recent Advances in Clinical Psychiatry 5 (ed. K. GranvilleGrossman) pp. 23-43. London: Churchill Livingstone

Lipsey, M. \& Wilson, D. (1993) The efficacy of psychological, educational and behavioural treatment: confirmation from meta-analysis. American Psychologist, 48, 1181-1209.

Malan, D. (1976) Individual Psychotherapy and the Science of Psychodynamics, pp. 79-80. London: Butterworth.

Marks, I. (1981) Cure and Care of Neuroses. Chichester: Wiley.

Orlinsky, D. \& Howard, K. (1993) Process and outcome in psychotherapy. In Handbook of Psychotherapy and Behaviour Change (eds A. E. Bergin \& S. L. Garfield), pp. 311-381. New York: Wiley.

Parry, G. \& Richardson, A. (1996) NHS Psychotherapy Services in England: Review of Strategic Policy. London: HMSO.

Roth, A. \& Fonagy, P. (1996) What Works for Whom? A Critical Review of Psychotherapy Research. New York: Guilford Press.

Shapiro, D. A. \& Shapiro, D. (1982) Meta-analysis of comparative outcome studies: $A$ replication and refinement. Psychological Bulletin, 92, 581-605.

Smith, M. L. \& Glass, G. V. (1977) Meta-analysis of psychotherapy outcome studies. American Psychologist, 32, 752-760.

- - \& Miller, T. (1980) The Benefits of Psychotherapy. Baltimore, MD: Johns Hopkins University Press.

Stevenson, J. \& Meares, R. (1992) An outcome study of psychotherapy for patients with borderline personality disorder. American Journal of Psychiatry, 149, 358-362.

Svartberg, M. \& Stiles, T. (1991) Comparative effects of short term psychodynamic psychotherapy: a meta-analysis. Journal of Consulting and Clinical Psychology, 59, 704-714.

Tillett, R. (1996) Psychotherapy assessment and treatment selection. British Journal of Psychiatry, 168, 10-15.

Williams, J. M. G. (1992) The Psychological Treatment of Depression (2nd edn). London: Routledge.

Yalom, I. (1985) The Theory \& Practice of Group Psychotherapy, pp. 3-18. New York: Basic Books.

\section{Multiple choice questions}

1. Existing psychotherapy research suggests that:

a most types of therapy are effective

b family intervention in schizophrenia increases relapse/readmission rate

c borderline personality disorder is untreatable

d there is no clear evidence of differential efficacy between therapies.
2. Assessment:

a should usually be completed at one interview

b should not normally include relatives' opinions

c should focus on childhood experience rather than current circumstances

d should include an appraisal of previous treatments.

3. A formulation:

a should be couched in terms of a particular theoretical model

b should identify who is to blame

c should not be discussed with the patient

d leads naturally towards a treatment proposal.

4. In planning treatment one should:
a avoid using medical and psychological approaches simultaneously
b use the least necessary treatment
c be decisive if the patient is hesitant
d agree a provisional treatment contract.

5. According to the Department of Health's report (Parry \& Richardson, 1996), psychotherapy services in the UK are:

a evenly distributed

b able to provide adequate training for junior psychiatrists

c affected by inter-disciplinary rivalries

d well-resourced.

\section{MCQ answers}

\begin{tabular}{|c|c|c|c|c|}
\hline 1 & 2 & 3 & 4 & \\
\hline a $T$ & a F & a $T$ & & F \\
\hline b F & b F & b F & b & $T$ \\
\hline F & c $F$ & c $F$ & & F \\
\hline d $\mathbf{T}$ & d $T$ & d $\mathrm{T}$ & d & $T$ \\
\hline
\end{tabular}

